\title{
Efeitos da suplementação dietética com cogumelos Agaricales e outros fungos medicinais na terapia contra o câncer
}

Effects of dietary supplementation with agaricales mushrooms and other medicinal fungus on therapy against the cancer

Renata Costa Fortes', Maria Rita Carvalho Garbi Novaes²

\section{Resumo}

Os cogumelos Agaricales têm sido utilizados, durante milênios, devido aos seus componentes biologicamente ativos que exercem efeitos nutricionais, medicinais e farmacológicos imprescindíveis para os portadores de diversos tipos de câncer. O objetivo deste estudo foi revisar os artigos indexados nas bases de dados Medline, Lilacs, NCBI, Capes, Scielo e Cochrane, que avaliaram os efeitos da suplementação dietética com cogumelos Agaricales e outros fungos medicinais na terapia contra o câncer.

Palavras-Chave: Agaricales, Cogumelos medicinais, Neoplasias, Suplementação dietética.

\section{Abstract}

The Agaricales mushrooms have been used during millennia due to its bioactive compounds that exert nutritional, medicinal and pharmacological effects that are essential for the patients of diverse types of cancer. The aim of this study is to revise indexed articles in the Medline, Lilacs, NCBI, Capes, Scielo e Cochrane databases, that had evaluated the effects of the dietary supplementation with Agaricales medicinal mushrooms and other fungus in the therapy against the cancer.

Key words: Agaricales, Medicinal mushrooms, Neoplasms, Dietary supplementation.

${ }^{1}$ Professora de Nutrição Humana da Faculdade de Ciências e Educação Sena Aires/GO. Preceptora da Residência em Nutrição Clínica da Secretaria de Saúde do Distrito Federal. Especialista em Clínica e Terapêutica Nutricional pelo Instituto Ponto Crítico de Ensino. Mestranda em Nutrição Humana da Universidade de Brasília.

${ }^{2}$ Professora Doutora da Faculdade de Medicina da Escola Superior em Ciências da Saúde/ DF. Professora Orientadora da Pós-Graduação em Nutrição Humana da Universidade de Brasília. Coordenadora do Comitê de Ética em Pesquisa da Secretaria de Saúde do Distrito Federal. Presidente da Sociedade Brasileira de Farmácia Hospitalar.

Enderę̧o para correspondência: QI 14. CJ J. CS 26. Guará 1/DF. CEP.: 71.015-100. E-mail: renatacfortes@yahoo.com.br - Telefone: (61) 9979-9463. 


\section{INTRODUÇÃO}

O câncer, resultado do acúmulo de mutações seqüenciais múltiplas e alterações moleculares que culminam com metástases ${ }^{1}$, é a segunda principal causa de mortalidade no Brasil ${ }^{2}$. Na França, o câncer é a maior causa de óbitos em homens e mulheres, entre 35 a 64 anos, sendo responsável por cerca de 150 mil mortes por ano $^{3}$. Nos Estados Unidos, estatísticas sugerem que, aproximadamente, $30 \%$ dos norte-americanos desenvolverão câncer durante toda a vida, sendo que, destes, dois terços morrerão em virtude dessa doençá.

Estimativas para o ano de 2006 indicam que ocorrerão, no Brasil, 472.050 casos novos de câncer, esperando-se 234.570 casos novos para o sexo masculino e 237.480 para o sexo feminino. Dados estatísticos apontam que o câncer de pele não-melanoma será o mais incidente na população brasileira (116 mil casos novos), seguido pelos tumores da mama (49 mil), próstata (47 mil), pulmão (27 mil), colorretal (25 mil), gástrico (23 mil) e colo do útero $(19 \mathrm{mil})^{5}$.

O desenvolvimento do câncer resulta da interação entre fatores endógenos e ambientais ${ }^{6}$. Cerca de 35\% dos diversos tipos de câncer ocorrem em decorrência de dietas inadequadas ${ }^{7}$, caracterizadas pelo alto teor de gordura saturada, colesterol e açúcares e baixo aporte de verduras, frutas, legumes e cereais integrais ${ }^{8}$. Tabagismo, obesidade, pouca atividade física, exposição a determinados tipos de vírus, bactérias, parasitas, além do contato freqüente com substâncias carcinogênicas também merecem ser destacados ${ }^{7}$.

Os tumores malignos, particularmente aqueles cujo crescimento é lento, levam maior tempo para serem diagnosticados, promovendo, conseqüentemente, alterações catabólicas extenuantes no hospedeiro, culminando com a caquexia ${ }^{8}$.

A caquexia, desnutrição em portadores de câncer, apresenta uma incidência entre 30 e 50\% dos casos, podendo estar associada ao aumento da morbimortalidade pós-operatória e menor tolerância aos procedimentos quimioterápicos, radioterápicos e cirúrgicos ${ }^{9}$.

$\mathrm{O}$ interesse no uso dos cogumelos e/ou de seus extratos como suplementos dietéticos vem crescendo significativamente, devido aos seus efeitos antitumorais, anticarcinogênicos, antivirais, antiinflamatórios, hipoglicemiantes, hipocolesterolêmicos, hipotensivos ${ }^{10}$, entre outros, podendo ser indicados como coadjuvantes no tratamento das neoplasias malignas ${ }^{8}$.

Considerando-se a relevância do tema, este estudo teve como objetivo principal investigar na literatura os efeitos nutricionais e farmacológicos dos cogumelos Agaricales e outros fungos medicinais na terapia contra o câncer.

\section{METODOLOGIA}

O presente artigo consiste em uma revisão crítica, sobre o tema, de artigos publicados principalmente em revistas indexadas nas bases de dados Medline, Lilacs, NCBI, Capes, Scielo e Cochrane, com ênfase nos últimos dez anos, nos idiomas inglês, espanhol e português, utilizando-se os termos Agaricales, cogumelos medicinais, câncer e suplementação dietética. Foram selecionados estudos experimentais em animais e ensaios clínicos randomizados, controlados, duplo-cegos, seguidos de tratamento estatístico com significância de $\mathrm{P}<0,05$.

\section{ALteRAÇÕES METABÓLICAS NO CÂNCER}

O comprometimento do estado nutricional nos pacientes com câncer está intimamente relacionado a índices elevados de morbimortalidade. Além da redução da ingestão protéico-calórica decorrente da diminuição do apetite, alterações do paladar ou comprometimento das funçōes orgânicas, diversas alteraçôes metabólicas contribuem para o desenvolvimento da caquexia observada nesses pacientes ${ }^{11}$.

As principais alterações metabólicas induzidas pelos tumores avançados incluem intolerância à glicose, redução da secreção de insulina, resistência periférica à insulina, aumento na síntese e no turn over de glicose, maior atividade do ciclo de Cori, aumento do turn over protéico, aumento na síntese hepática de proteínas, aumento no catabolismo protéico muscular, redução plasmática da concentração de aminoácidos ramificados, depleção dos depósitos lipídicos, aumento da lipólise, aumento do turn over de glicerol e ácidos graxos livres, redução da lipogênese e hiperlipidemia ${ }^{11}$.

Os mecanismos gerais relacionados ao processo de carcinogênese envolvem a secreção de citocinas, hormônios reguladores e contra-reguladores. As principais citocinas e hormônios que participam desse processo são fatores de necrose tumoral (TNF- $\alpha$ ), interleucina 1 (IL-1), interleucina 2 (IL-2), interleucina 6 (IL-6), interferon gama (ITF- $\gamma$ ), glucagon, cortisol, catecolaminas e hormônio de crescimento ${ }^{11,12}$. A serotonina, leptina, fator de mobilização lipídica, fator de mobilização protéica também estão envolvidos no processo relacionado à caquexia do câncer através de diversas atividades biológicas ${ }^{12}$.

Além dessas alterações, o próprio tratamento convencional do câncer exibe diversos graus de 
desnutrição, devido às complicações e/ou efeitos colaterais como diarréia, náuseas, vômitos, mucosites e anorexia, diminuindo significativamente a absorção dos nutrientes, tornando o paciente cada vez mais susceptível às infecçôes ${ }^{13}$.

\section{ProcesSO de FORMAÇÃO DO CÂNCER E ATUAÇÃO dOS FATORES DIETÉTICOS NOS ESTÁGIOS DE CARCINOGÊNESE}

O processo de carcinogênese pode ser dividido em três estágios: iniciação, promoção e progressão. A fase de iniciação envolve a exposição aos carcinógenos e danos nas moléculas de DNA. Na fase de promoção, os promotores tumorais ou mitógenos ativos induzem a expansão clonal das células iniciadas. $\mathrm{Na}$ fase de progressão, as células alteradas desenvolvem modificações irreversíveis, resultando na proliferação descontrolada de células cancerosas ${ }^{1,14}$.

A dieta adequada exerce um papel crucial nos estágios de iniciação, promoção e progressão do câncer, podendo prevenir de três a quatro milhôes de casos novos de câncer a cada ano ${ }^{7}$. Determinados fungos medicinais contêm componentes capazes de modular a tumorigênese e carcinogênese nos diferentes estágios da doença e/ou agir em um mesmo estágio através de diferentes mecanismos, exercendo, dessa forma, efeitos benéficos na prevenção e no tratamento do câncer ${ }^{14}$.

\section{Propriedades Terapêtuticas dos Cogumelos aGAPICAESE OUTROS FUNGOS MEDICINAIS}

Os cogumelos eram conhecidos pela humanidade pré-histórica por suas propriedades nutricionais e medicinais, além da toxicidade de algumas espécies. Atualmente, o consumo dos cogumelos continua sendo bastante apreciado em distintas culturas devido às suas características organolépticas ${ }^{15}$. Existem, no mínimo, 10.000 espécies de cogumelos, sendo 700 comestíveis, 50 a 200 medicinais e 50 venenosas 8 .

A ordem Agaricales, família Agaricaceae, é uma das mais numerosas, importantes e estudadas, devido às suas propriedades farmacológicas, inclusive as do gênero Agaricus, porém também possui algumas espécies tóxicas (amanitinas) e alucinógenas (alcalóides psilocibinas) ${ }^{15}$.

As quatro espécies da ordem Agaricales que mais se destacam na indústria de alimentos, devido ao elevado cultivo, são Agaricus bisporus ou champignon de Paris, Lentinus edodes ou shiitake, Pleurotus ostreatus ou cogumelo ostra e Volvariella volvaceae ou fukurotake ${ }^{15}$.

No Brasil, os cogumelos de interesse comercial são encontrados nas seguintes espécies: Agaricus bisporus,
Lentinus edodes, Pleurotus ostreatus, Agaricus blaze ${ }^{16}$, Agaricus brasiliensis ${ }^{17}$ e Agaricus sylvaticus, que possuem diferenças no genótipo ou ainda no fenótipo das espécies $^{18}$.

Os cogumelos são considerados alimentos nutracêuticos, apresentando excelente eficácia quando consumidos como suplementos dietéticos e podem ser utilizados como fármacos através da extração dos princípios ativos, assim como produzidos quimicamente pela indústria farmacêutica ${ }^{8,19}$.

Atualmente, entre os compostos derivados dos cogumelos, são comercializados diversos produtos, tais como: Cogumelo do sol ${ }^{\circledR}$ (Agaricus sylvaticus), Agaricus JUN-17® (Agaricus blazei), Lentinan ${ }^{\circledR}$ (Lentinus edodes), Krestin ${ }^{\circledR}$ (Trametes versicolor), Schizophyllan ${ }^{\circledR}$ (Schizophylum communis), Grifron ${ }^{\circledR M a i t a k e ~(G r i f o l a ~}$ frondosa), Reishi ${ }^{\circledR}$ (Ganoderma lucidum), entre outros.

\section{COMPOSIÇÃO QUÍMICA VERSUS VALOR NUTRITIVO}

Determinados tipos de cogumelos são indicados para dietas hipoenergéticas ou como coadjuvantes no tratamento de enfermidades especiais como o câncer. Podem ser consumidos sob as formas desidratada e fresca, diferindo apenas no teor hídrico e na apresentação com conservantes ${ }^{15}$.

Quimicamente e nutricionalmente, os cogumelos são considerados alimentos saudáveis ${ }^{20}$, pois apresentam características imprescindíveis para a execução de suas funçôes. A tabela 1 ilustra a composição nutricional de algumas espécies de cogumelos ${ }^{21,22}$.

Tabela 1. Composição nutricional de algumas espécies de cogumelos

\begin{tabular}{lc}
\hline \multicolumn{1}{c}{ Nutrientes } & Porcentagem (\%) \\
\hline Água & 90 \\
Carboidrato & $03-28$ \\
Proteína & $10-40$ \\
Lipídeo & $02-08$ \\
Fibras & $03-32$ \\
Minerais & $08-10$ \\
\hline
\end{tabular}

Fonte: Adaptado de Breene ${ }^{21}$ e Borchers et al. ${ }^{22}$

Os cogumelos possuem teores elevados de carboidratos, fibras, $\beta$-glucanas, $\beta$-proteoglucanas, heteroglicanas, quitina e peptideoglucanas ${ }^{8}$. Com base em peso seco, os teores glicídicos variam de 51 a $88 \%$, estando presentes nas cadeias $ß$-glucanas, nas paredes celulares e nas regiōes intracitoplasmáticas ${ }^{15}$. 
As fibras dietéticas estão contidas nos cogumelos em proporçôes que variam de 10 a 50\% com base em peso seco e possuem ação física desfavorável na absorção de substâncias tóxicas, nocivas e carcinogênicas. Inúmeras pesquisas demonstram que as fibras estão associadas a uma menor incidência de câncer colorretal, uma vez que aceleram a excreção do bolo alimentar por ação mecânica laxativa, diminuindo o tempo de permanência intestinal ${ }^{15}$.

Comparando-se o teor protéico da carne bovina com algumas espécies Agaricales, observa-se que a carne bovina possui em torno de $14,8 \%$ de proteína em peso seco, ao passo que os fungos Agaricales apresentam $22,5 \%{ }^{15}$. Além do alto teor protéico, os cogumelos são considerados proteína de alto valor biológico, uma vez que possuem todos os aminoácidos indispensáveis ${ }^{23}$, além de arginina, glutamina, dentre outros ${ }^{24}$. Segundo Mdachi et al. ${ }^{25}$, os diferentes tipos de aminoácidos variam em números de seis a 15 , dependendo da espécie, tornando-os comparáveis em termos nutritivos com as carnes, os ovos e o leite.

Apesar de apresentarem quantidades reduzidas de gorduras totais, possuem alta porcentagem de ácidos graxos poliinsaturados (PUFA) e baixos teores de ácidos graxos saturados e colesterol ${ }^{22}$. Comparando-se com os peixes e as aves, os cogumelos apresentam quantidades bem menores de colesterol e gordura saturada. A gordura bruta dos cogumelos é constituída por diversas classes de lipídeos, incluindo os ácidos graxos livres, mono-ditriglicérides, esteróis, terpenóides e fosfolipídeos, destacando-se a lecitina ${ }^{15}$.

Os ácidos graxos poliinsaturados são conhecidos pelo importante papel no desenvolvimento e homeostase normais. Estudos epidemiológicos demonstram que populações com maior ingestão de PUFA possuem redução significativa na incidência e mortalidade por câncer quando comparadas com populações com menor ingestão desses nutrientes ${ }^{26}$.

Os cogumelos possuem quantidades significativas de potássio, cálcio, fósforo, magnésio, ferro, zinco ${ }^{22}$, sódio $^{23}$, niacina, tiamina, riboflavina, biotina, ácido ascórbico e pró-vitaminas A e D (ergosterol) ${ }^{22}$.

Metais pesados como arsênico, cádmio, mercúrio ${ }^{14}$, chumbo e cobre podem estar presentes, principalmente quando não houver adequado cuidado nas áreas de cultivo e com a água utilizada ${ }^{15}$, assim como substâncias radioativas como ${ }^{137} \mathrm{Cs}^{14}$.

\section{AÇÃO DAS PRINCIPAIS SUBSTÂNCIAS BIOATIVAS DOS COGUMELOS COM EFEITOS FARMACOLÓGICOS}

Evidências científicas têm demonstrado que as $\beta$ glucanas exercem atividades antitumorais ${ }^{24}$; as $\beta$ proteoglucanas, atividades antitumoral ${ }^{19}$, antiviral ${ }^{27} \mathrm{e}$ antitrombocítica ${ }^{28}$; a lecitina exerce propriedade antitumoral $^{29}$, antimutagênica ${ }^{30}$ e hemaglutinizante ${ }^{3}$; o ergosterol funciona como anticarcinogênico ${ }^{32}$ e inibidor da angiogênese ${ }^{33}$; o ácido linoléico como bactericida; os esteróides atuam contra os tumores ${ }^{19}$; arginina como anticarcinogênica $^{24}$ e glutamina com efeitos antioxidantes, entre outros ${ }^{34}$.

Os cogumelos são capazes de modular a carcinogênese em todos os estágios da doença através de distintos mecanismos ${ }^{14}$. Porém, os mecanismos de ação dos princípios ativos presentes nos cogumelos Agaricales e em outros fungos medicinais ainda não estão completamente esclarecidos na literatura. Investigadores sugerem que essas ações podem ser atribuídas a componentes específicos destes fungos, destacando-se as glucanas, o ergosterol, as lecitinas e alguns aminoácidos imunomoduladores como a arginina e a glutamina ${ }^{15}$. A tabela 2 ilustra a atuação dessas substâncias bioativas e o seu provável mecanismo de ação.

\section{ESTUDOS EXPERIMENTAIS COM COGUMELOS AGARICAESE OUTROS FUNGOS MEDICINAIS}

Estudos experimentais demonstraram que a administração intraperitoneal de grifolana (Grifola frondosa), em ratos com sarcoma 180 (sólido), nas dosagens de 20, 100 e $200 \mathrm{mg}$, promoveu inibição tumoral superior a 38 e $99 \%$, respectivamente ${ }^{44}$.

Extratos solúveis em água de Agaricus blazei testados em ratos Swiss machos com genotoxicidade induzida por ciclofosfamida nas concentraçôes de $4^{\circ} \mathrm{C}, 21^{\circ} \mathrm{C}$ e $60^{\circ} \mathrm{C}$ promoveram inibição significativa da indução de micronúcleo pela ciclofosfamida na medula espinhal e no sangue periférico dos ratos in vivo, demonstrando que a atividade antimutagênica pode contribuir para o efeito anticarcinogênico ${ }^{45}$.

A administração oral de frações solúveis em água quente do complexo protéico $\alpha(1,6)$ glucana e $\alpha(1,4)$ glucana extraídas de Agaricus blazei em ratos com sarcoma 180 resultou em redução tumoral significativa $(\mathrm{P}<0,05)^{46}$.

Outro estudo demonstrou que a administração de $10 \mathrm{mg} / \mathrm{kg} / \mathrm{dia}$ do polissacarídeo lentinana (Lentinus edodes) e de $200 \mathrm{mg} / \mathrm{kg} /$ dia do polissacarídeo Agaricus blazei (ABPS) em ratos Balb fêmeas com carcinoma induzido por 3-metilcolantrene promoveu supressão do 
Tabela 2. Provável mecanismo de ação de algumas substâncias contidas nos cogumelos

\begin{tabular}{|c|c|c|}
\hline Substância & Ação & Provável Mecanismo \\
\hline Glucana & $\begin{array}{c}\text { Inibe o crescimento tumoral }{ }^{35} \text {; promove } \\
\text { ativação de células Natural Killer (NK), } \\
\text { linfócitos T e B, células complementares; } \\
\text { aumenta o número de macrófagos }{ }^{36} \text {, } \\
\text { monócitos, anticorpos, IL-2, IL-6, IFN- } \gamma \text { e } \\
\text { TNF- } \alpha^{37} \text {. }\end{array}$ & $\begin{array}{c}\text { Ativação e expansão clonal de células T. A } \\
\text { interação de células T com antígenos inicia uma } \\
\text { cascata de eventos bioquímicos e expressão } \\
\text { genética, induzindo o restante das células Ta } \\
\text { entrarem no ciclo celular iniciando os processos de } \\
\text { proliferação e diferenciação }{ }^{19} \text {. }\end{array}$ \\
\hline Ergosterol & $\begin{array}{c}\text { Redução do volume e inibição do } \\
\text { crescimento tumoral, em ratos com sarcoma } \\
180, \text { via oral e intraperitoneal, } \\
\text { respectivamente, sem efeitos adversos } \\
\text { geralmente causados pelos agentes } \\
\text { quimioterápicos }{ }^{33} \text {. }\end{array}$ & $\begin{array}{l}\text { Inibição da neovascularização induzida pelo } \\
\text { crescimento tumoral nas células do sarcoma } 180 \\
\text { in vitro. O ergosterol (precursor do ergocalciferol) } \\
\text { é uma substância antiangiogênica explicando, em } \\
\text { parte, o seu efeito antitumoral }{ }^{33} \text {. }\end{array}$ \\
\hline Lecitina & $\begin{array}{l}\text { Age contra células: sarcoma 180, tumoral } \\
\text { humana, cancerosa colônica humana e do } \\
\text { câncer mamário. Inibe o crescimento de } \\
\text { células do mastocitoma in vitro e do } \\
\text { sarcoma } 180 \text { in vivo em ratos }{ }^{38} \text {. }\end{array}$ & $\begin{array}{c}\text { Propriedade indutora de apoptose nas células } \\
\text { tumorais - mecanismo primário contra as } \\
\text { neoplasias malignas }{ }^{38} \text {. }\end{array}$ \\
\hline $\begin{array}{l}\text { Proteo- } \\
\text { glucanas }\end{array}$ & $\begin{array}{c}\text { Aumento significativo da sobrevida de } \\
\text { pacientes com câncer nasofaríngeo, } \\
\text { esofágico, gástrico, colorretal, pulmonar e } \\
\text { mamário }^{39} \text { e de ratos radiados. Melhora da } \\
\text { qualidade de vida, alívio dos sintomas em } \\
70 \text { a } 97 \% \text { dos pacientes com câncer } \\
\text { gástrico, esofágico, pulmonar, ovariano e } \\
\text { cervical }{ }^{19} \text {. }\end{array}$ & $\begin{array}{l}\text { Estimulação das funções imunológicas, da } \\
\text { atividade fagocitária dos macrófagos e melhoria } \\
\text { das funções do sistema retículo-endotelial }{ }^{19} \text {. }\end{array}$ \\
\hline Arginina & $\begin{array}{l}\text { Inibição do crescimento tumoral }{ }^{40} \text {; redução } \\
\text { significativa dos casos de perdas } \\
\text { nitrogenadas em pacientes com trauma } \\
\text { cirúrgico, via parenteral; redução do tempo } \\
\text { de internação hospitalar de pacientes no } \\
\text { pós-operatório de cirurgias de grande porte } \\
\text { por câncer, via enteral }{ }^{41} \text {. }\end{array}$ & $\begin{array}{c}\text { Aumento da atividade das células NK e T Helper. } \\
\text { Estímulo das sínteses de IL-1, IL-2, IL-6, TNF- } \alpha^{42} \text {. } \\
\text { Pode promover aumento da imunidade através da } \\
\text { liberação do hormônio de crescimento, estímulo } \\
\text { na produção de óxido nítrico, hidroxiprolina, } \\
\text { citocinas e poliaminas }{ }^{41} \text {. }\end{array}$ \\
\hline Glutamina & $\begin{array}{c}\text { Aumento da função imune e intestinal; } \\
\text { redução da bacteremia e dos danos na } \\
\text { mucosa associados à quimioterapia, } \\
\text { manutenção da integridade intestinal após } \\
\text { quimioterapia e radioterapia }{ }^{43} \text {. Maior } \\
\text { preservação do músculo esquelético; } \\
\text { melhora do equilíbrio nitrogenado; } \\
\text { nenhuma elevação de citocinas pró- } \\
\text { inflamatórias e capacidade antioxidante } \\
\text { (glutationa) }{ }^{34} \text {. }\end{array}$ & $\begin{array}{l}\text { A glutamina é a fonte de energia preferencial à } \\
\text { glicose por todas as células de rápida divisão, } \\
\text { como os enterócitos e células do sistema } \\
\text { imunológico, assim como para o sistema nervoso } \\
\text { e como coadjuvante no tratamento de câncer } \\
\text { avançado, prolongando a sobrevida e diminuindo } \\
\text { o catabolismo debilitante, além de promover uma } \\
\text { maior tolerância à quimioterapia }{ }^{43} \text {. }\end{array}$ \\
\hline
\end{tabular}

citocromo P450s para 66\% (lentinana) e para $73 \%$ $(\mathrm{ABPS})^{47}$.

Segundo Wasser ${ }^{48}$, a carcinogênese foi investigada após a indução de carcinógenos em ratos e administração, no grupo controle, de uma dieta contendo 5\% de Hypsizygus marmoreus (cogumelo seco). Ao final de 76 semanas, constatou-se que 21 dos 36 ratos não tratados desenvolveram tumor, ao passo que apenas três dos 36 ratos que receberam o tratamento com Hypsizygus marmoreus apresentaram o desenvolvimento tumoral, demonstrando que tanto o mecanismo inibitório quanto a atividade preventiva do câncer por atuação dos cogumelos se deve à imunopotenciação.

Em ratos submetidos a injeções subcutâneas de chá de Agaricus sylvaticus preparado a $100^{\circ} \mathrm{C}$, observou-se que os números de células NK triplicaram no exsudato peritoneal, fato não observado no grupo controle. Para cada 12 células NK do controle e do Agaricus sylvaticus, 
averiguou-se, em três horas de incubação, $1 \%$ e $40 \%$ de inibição de células leucêmicas, respectivamente. Para cada 25 células NK, observou-se inibição de 1\% (controle) e de $48 \%$ (Agaricus sylvaticus) e para cada 50 células NK houve inibição das células leucêmicas de 1,5\% (controle) e 57\% (Agaricus sylvaticus) ${ }^{49}$.

Novaes et al. ${ }^{18}$ avaliaram os efeitos da administração de $50 \mathrm{mg} / \mathrm{kg} / \mathrm{dia}$ de extratos de Agaricus sylvaticus em ratos Wistar com tumor ascítico Walker 256 e observaram melhora significativa das funções hematológicas e imunológicas. Segundo Sorimachi et al. ${ }^{50}$, acredita-se que o provável mecanismo de ação consiste na inibição do crescimento tumoral e na estimulação dos sistemas hematológicos e imunológicos.

Em um estudo randomizado, duplo-cego e placebocontrolado, 46 ratos machos com tumor Walker $256 \mathrm{e}$ anemia severa foram divididos em dois grupos: placebo $(n=43)$ e experimental $(n=43)$. O grupo experimental recebeu uma dieta contendo $50 \mathrm{mg} / \mathrm{kg} / \mathrm{dia}$ de extratos aquosos de Agaricus sylvaticus por gavagem, duas vezes ao dia, até a morte. $\mathrm{O}$ grupo experimental apresentou aumento significativo dos eritrócitos $(P=0,03)$, hematócrito $(P=0,02)$, hemoglobina $(P=0,05)$, leucócitos, linfócitos e neutrófilos $(P=0,03)$, demonstrando que a suplementação com a Agaricus sylvaticus exerce efeitos benéficos em ratos com tumor Walker 256, principalmente, no sistema hematopoiético ${ }^{51}$.

\section{ENSAIOS CLÍNICOS COM COGUMELOS AGAPICAESE OUTROS FUNGOS MEDICINAIS}

Em um ensaio clínico randomizado, placebocontrolado e duplo-cego, 20 pacientes com câncer colorretal, em fase pós-operatória, tratados com quimioterapia, foram divididos em dois grupos: experimental $(n=10)$ - suplementado com fungo Agaricus sylvaticus, oralmente, duas vezes ao dia $(4,08 \mathrm{mg} / \mathrm{kg} / \mathrm{dia})$ e placebo $(n=10)$ - apenas amido, oralmente. Todos os pacientes foram acompanhados por um período de três meses. O grupo suplementado com Agaricus sylvaticus apresentou redução dos níveis de colesterol total de $249,60 \pm 119,62$ para $206,80 \pm 24,46(P=0,01)$, fato não observado no grupo placebo. Os níveis de LDL-c reduziram no grupo experimental, porém esta redução não foi estatisticamente significativa. Níveis de HDL e VLDL não alteraram em ambos os grupos. As taxas de triglicerídeos foram maiores no grupo placebo $(P=0,05)$ quando comparado com o grupo tratado, demonstrando que a suplementação com Agaricus sylvaticus pode beneficiar esses pacientes através da regulação do metabolismo lipídico ${ }^{52}$.

Os efeitos de lentinana (Lentinus edodes), durante seis meses, foram avaliados em 33 pacientes em vários estágios de câncer gástrico após gastrectomia. Após uma semana, esses pacientes receberam $2 \mathrm{mg}$ de lentinana, quatro vezes ao dia, por dois ou quatro intervalos semanais. Os resultados obtidos foram aumento superior a $50 \%$ da produção basal de IL-1 pelos macrófagos em aproximadamente $70 \%$ dos pacientes. Este efeito foi mais significativo nos pacientes que receberam lentinana por quatro semanas quando comparado com aqueles que receberam esse tratamento por apenas duas semanas ${ }^{22}$.

A administração de $3 \mathrm{~g} /$ dia de polissacarídeopeptídeo (PSP) extraído do micélio de Coriolus versicolor, por um período de dois meses, em 82 pacientes com carcinoma gástrico tratados com quimioterapia, resultou em aumento significativo $(P<0,05)$ da atividade das células NK, IL-2, CD4, CD8 quando comparado com o grupo controle que recebeu apenas o tratamento quimioterápico ${ }^{53}$.

Em um ensaio clínico randomizado, placebocontrolado e duplo-cego, 68 pacientes com câncer pulmonar avançado (estadiamentos III e IV) que haviam terminado o tratamento convencional, foram divididos em dois grupos: placebo $(n=34)$ e experimental $(n=$ 34). O grupo experimental foi suplementado, via oral, com três cápsulas de PSP isolado de Coriolus versicolor (340 mg cada), três vezes ao dia, durante quatro semanas. Observou-se aumento significativo $(P<0,05)$ nas contagens de leucócitos e neutrófilos, enquanto no grupo placebo essas taxas foram reduzidas. Os níveis de $\operatorname{IgG}$, IgM e o percentual de gordura corpórea aumentaram significativamente $(P<0,05)$ no grupo experimental, fato não observado no grupo placebo. Embora os pacientes do grupo PSP não tenham apresentado melhora significativa dos sintomas, observou-se que o número de pacientes que abandonaram o estudo, devido à progressão da doença, foi significativamente maior no grupo placebo $(n=8)$ quando comparado com o grupo tratado $(n=2)(P=0,04)$. Nenhuma reação adversa foi atribuída aos medicamentos experimentais. Os resultados sugerem que a ingestão de PSP pode tornar mais lento o processo de deterioração da doençç ${ }^{54}$.

Duzentos e sessenta e cinco pacientes com carcinoma gástrico em tratamento cirúrgico e quimioterápico foram randomizados em dois grupos: placebo (tratado apenas com quimioterapia) e experimental (quimioterapia mais $3 \mathrm{~g} /$ dia de polissacarídeo, PSK, de Coriolus versicolor), durante um período de quatro semanas que foram alternadas com um período de repouso semanal. O grupo tratado com PSK demonstrou aumento significativo $(P$ $<0,05)$ da sobrevivência por cinco anos e na taxa de sobrevida geral. Em relação aos efeitos adversos, não 
houve diferença significativa entre os dois grupos ${ }^{55}$.

Em outro estudo, 20 pacientes com leucemia nãolinfocítica aguda tratados com quimioterapia foram assim divididos: grupo experimental (20g de Agaricus blazei, três vezes ao dia; $n=10$ ) e grupo controle (placebo; $n=$ 10). No grupo experimental, 8 pacientes alcançaram completa remissão tumoral, 2 pacientes permaneceram sem remissão e a taxa entre eritrócitos, granulócitos e grandes células nucleares retornaram aos níveis normais num período de sete a oito dias ao final da quimioterapia. No grupo controle, 5 pacientes alcançaram completa remissão, 2 pacientes tiveram remissão parcial e 3 não apresentaram remissão do tumor. Observou-se também aumento significativo nas quantidades de $\operatorname{IgM}$ no grupo que recebeu o cogumelo, porém não foram detectadas modificações no grupo controle ${ }^{56}$.

Cinqüenta pacientes com câncer gástrico tratados com cirurgia e quimioterapia participaram de um ensaio clínico randomizado, duplo-cego, placebo controlado e receberam, por um período de três meses, $3 \mathrm{~g} / \mathrm{dia}$ de PSP (Coriolus versicolor) ou placebo. Os resultados obtidos foram aumento significativo $(P<0,05) \mathrm{da}$ atividade das células NK e de CD4/CD8 quando comparado com o grupo controle ${ }^{57}$.

Quinze mulheres com carcinoma mamário em estágio avançado da doença receberam D-fração- $\beta$ glucana e tabletes de maitake extraídos de Grifola frondosa aliados à quimioterapia e $86,7 \%$ obtiveram como resultados redução significativa do tamanho tumoral, melhoria nos testes séricos, redução dos vômitos e aumento do apetite . $^{58}$.

Em outro estudo clínico randomizado, placebocontrolado e duplo-cego, 22 pacientes foram divididos em dois grupos (placebo, $n=11$; suplementado com Agaricus sylvaticus, $n=11)$. $\mathrm{O}$ grupo Agaricus sylvaticus recebeu o cogumelo via oral $(4,08 \mathrm{mg} / \mathrm{kg} / \mathrm{dia}$, duas vezes ao dia, por três meses). O grupo placebo recebeu somente amido. Dos 11 pacientes do grupo placebo, 9\% apresentaram constipação, 27\%, diarréia e 64\%, ausência de alterações após início da suplementação, sendo que, destes, 91\% relataram nenhuma modificação na quantidade e consistência fecal e apenas $9 \%$ relataram melhoria fecal. Comparando os resultados com o grupo Agaricus, observou-se que 91\% dos pacientes deste grupo experimentaram normalização intestinal, através da melhoria tanto da diarréia quanto da constipação e apenas $9 \%$ relataram não ter tido alterações na função intestinal. Observou-se que, no grupo placebo, 28\% dos pacientes apresentaram tontura, 27\%, dores abdominais, 18\%, insônia, 9\%, fraqueza e 18\%, ausência de alterações. Em comparação com o grupo Agaricus, 55\% apresentaram melhoria na disposição e
36\%, ausência de alterações nas funções intestinais. Os resultados demonstraram que a suplementação com Agaricus sylvaticus promove redução significativa dos efeitos quimioterápicos adversos, normalização das funções intestinais e melhora significativa da qualidade de vida em pacientes com câncer colorretal ${ }^{59}$.

\section{CONCLUSÃO}

Estudos clínicos e experimentais demonstram que a suplementação dietética com cogumelos Agaricales e outros fungos medicinais exerce efeitos nutricionais, medicinais e farmacológicos imprescindíveis, podendo ser utilizada como coadjuvante na terapia contra o câncer. Os mecanismos de ação das substâncias bioativas presentes nos cogumelos ainda não estão completamente esclarecidos na literatura, mas evidências científicas sugerem que essas substâncias são capazes de modular a carcinogênese nos estágios de iniciação, promoção e progressão, promovendo benefícios aos portadores de diversos tipos de câncer, principalmente através da estimulação do sistema imunológico. Estudos controlados e randomizados adicionais são necessários para elucidar detalhadamente os possíveis efeitos adversos, toxicidade e mecanismo de ação dos principais componentes bioativos presentes nos fungos medicinais.

\section{REFERÊNCIAS}

1. Gescher AJ, Sharma RA, Steward WP. Cancer chemoprevention by dietary constituents: a tale of failure and promise. Lancet Oncol. 2001;2:371-9.

2. Instituto Nacional de Câncer. Estimativa da incidência e mortalidade por câncer no Brasil 1999. Rio de Janeiro: INCA; 2000.

3. Ganry O, Boche T. Prevention practices and cancer screening among general practitioners in Picardy, France. Public Health. 2005;119:1023-30.

4. Andreoli TE, Bennett JC, Carpenter CCJ, Plum F. Doenças Neoplásicas. Cecil Medicina Interna Básica. Rio de Janeiro: Guanabara Koogan; 1998. p. 407-423.

5. Instituto Nacional de Câncer. Estimativa 2006: Incidência de câncer no Brasil. Rio de Janeiro: INCA; 2005.

6. Schieri R, Everhart JE, Mendonça GAS. Diet and mortality from common cancers in Brazil: an ecological study. Cad Saúde Pública. 1996;12(1):53-9.

7. Garófolo A, Avesani CM, Camargo KG, Barros ME, Silva SRJ, Taddei JAAC, et al. Dieta e câncer: um enfoque epidemiológico. Rev Nutr PUCCAMP. 2004;17(4):491-505.

8. Novaes MRCG, Fortes RC. Efeitos antitumorais de cogumelos comestíveis da família agaricaceae. Rev Nutr Brasil. 2005;4(4):207-217. 
9. Waitzberg DL. Câncer. In.: Waitzberg DL: Nutrição oral, enteral e parenteral na prática clínica. São Paulo: Atheneu; 2000, p. 1381-1393.

10. Miles PC, Chang ST. Mushroom biology: concise basics and current developments. Singapore: World Scientific; 1997.

11. Montagnini AL, Perini MV. Alterações metabólicas do câncer. In.: Ikemori EHA, Oliveira T, editores. Nutrição em oncologia. São Paulo: Editora Marina e Tecmedd; 2003. p. 35-44.

12. Ych SS, Schuster MW. Geriatric cachexia: the role of cytokines. Am J Clin Nutrition. 1999;70(2):183-97.

13. Matthys P, Billiau A. Cytokines and cachexia. Nutrition. 1997;13(9):763-69.

14. Borchers AT, Keen CL, Gershwin ME. Mushrooms, tumors, and immunity: an update. Exp Biol Med. 2004;229:393-406.

15. Novaes MRCG, Novaes LCG. Fármaco-nutrientes em cogumelos comestíveis Agaricales e outros basidiomicetos. Rev Bras Nutr Clin. 2005;20(3):181-7.

16. Urben A.F, Uriartt AH. Curso de cultivo de Lentinus edodes (shiitake) com "Juo-Cao". Rio de Janeiro: Embrapa; 2002. p. 84-105.

17. Didukh MY, Wasser SP, Nevo E. Medicinal value of species of the family agaricaceae cohn (higher basidiomycetes and future perspectives. Int J Med Mushrooms. 2003;5:133-52.

18. Novaes MRG, Garcez LCG, Melo A, Recova V. Effects of administration of Agaricus sylvaticus fungi on hematological and immunological systems of rats with walker-256 carcinoma. Fundam Clin Pharmacol. 2004;18(S1):125-9.

19. Kuo YC, Huang YL, Chen CC, Lin YL, Chuang KA, Tsai WJ. Cell cycle progression and cytokine gene expression of human peripheral blood mononuclear cells modulated by Agaricus blazei. J Lab Clin Med. 2002;140(3):176-87.

20. Dias ES, Abe C, Schwan RF. Truths and myths about the mushroom Agaricus blazei. Sci Agric. 2004;61(5):545-9.

21. Breene WM. Nutritional and medicinal value of speciality mushrooms. J Food Project. 1990;53:883-94.

22. Borchers AT, Stern JS, Hackman RM, Keen CL, Gershwin ME. Mushroom, tumors, and imumnity. PSEBM. 1999;221(4):281-93.

23. Amazonas Mala. Importância do uso de cogumelos: aspectos nutricionais e medicinais. Rio de Janeiro: Embrapa; 2002, p.143-161.

24. Mizuno T, Zhuang C. "Maitake Grifola frondosa, pharmacological effects". Food Rev Int. 1995;11(1):135-49.

25. Mdachi SJM, Nkunya MHH, Nyigo VA, Urasa IT. Amino acid composition of some Tanzanian wild mushrooms. Food Chem. 2004;86:179-82.

26. Roynette CE, Calder PC, Dupertuis YM, Pichard C. n-3 Polyunsaturated fatty acids and colon cancer prevention. Clin Nutr. 2004;23(2):139-51.

27. Kawagishi H. Erinacines A, B, C, strong stimulators of nerve growth factor synthesis, from the mycelia of Hericium erinaceum. Tetrahedron Lett. 1994;35(10): 1569-72.

28. Pai SH, Jong SC, Lo DW. Uses of mushrooms. Bioindustry. 1990;1:126-31.

29. Zhao C, Sun H, Tong X, Qi Y. School of life science, wuhan university, wuhan city, hubei province, 430072, people's republic of china. Biochem J. 2003; 374 (Pt 2): 321-7.

30. Ho JC, Sze SC, Shen WZ, Liu WK. Mitogenic activity of edible mushrooms lectins. Biochim Biophys Acta. 2004;1671(1-3):9-17.

31. Wang $\mathrm{H}, \mathrm{Ng}$ TB. Isolation of a novel $\mathrm{N}$-acetylglucosaminespecific lectin from fresh sclerotia of the edible mushroom Pleurotus tuber-regium. Protein Expr Purif. 2003;29(2):156-60.

32. Stamets P, Yao CDW. Mycomedicinals: na information booklet on medicinal mushrooms. Olympia: MycoMedia; 1998.

33. Takaku T, Kimura Y, Okuda H. Isolation of ana antitumor compound from Agaricus blazei Murril and its mechanism of action. J Nutr. 2001;131:1409-13.

34. Méier R, Steuerwald M, Waitzberg DL. Imunonutrição e câncer. In.: Waitzberg DL: Nutrição e Câncer. São Paulo: Atheneu; 2004. p. 630-35.

35. Mizuno T, Saito H, Nishitoba T, Kawagishi H. Antitumoractive substances from mushrooms. Food Rev Int. 1995;11:23-61.

36. Mizuno T. A development of antitumor polysaccharides from mushroom fungi foods food ingredients. Jpa J. 1996;167:69-85.

37. Chu KKW, Ho SSS, Chow AHL. Coriolus versicolor: a medicinal mushroom with promising immunotherapeutic values. J Clin Pharmacol. 2002; 42:976-84.

38. Zhao C, Sun H, Tong X, Qi Y. School of life science, wuhan university, wuhan city, hubei province, 430072, people's republic of china. Biochem J. 2003;374(Pt 2):321-7.

39. Kidd PM. The use of mushroom glucans and proteoglycans in cancer treatment. Altern Med Rev. 2000;5(1):4-27.

40. Novaes MRCG, Lima ABM. Efeitos da suplementação dietética com L-arginina no paciente oncológico: uma revisão da literatura. Arch Latino Americanos de Nutr. 1999; 49(4):301-8.

41. Novaes MRCG, Beal FL. Suporte nutricional com arginina: perspectivas terapêuticas em portadores de câncer. Rev SBRAFH. 2003;1:14-9.

42. Novaes MRCG, Lima LAM, Ribeiro JEG, Magalhães AV, Sousa MV, Morhy L. Efeitos farmacológicos da suplementação dietética com arginina a $6 \%$ em tumores experimentais. Rev Metab Nutr. 2003;7(2) 230-6.

43. Skubitz KM. Glutamine as a potential tratment for the prevention of chemotherapy - induced mucosistis. J Infus Chemother. 1994;4:64-7.

44. Ohno N, Adachi Y, Suzuki I, Sato K, Oikawa S, Yadomae T. Characterization of the antitumor glucan obtained from 
liquid-cultured Grifola frondosa. Chem Pharm Bull. 1986;34:1709-15.

45. Delmanto RD, Lima PLA, Sugui MM, Eira AF, Salvadori DMF, Speit G, et al. Antimutagenic effect of Agaricus blazei Murril mushroom on the genetoxicity induced by ciclophosphamide. Mutat Res. 2001;496(1-2):15-21.

46. Mizuno M, Morimoto M, Minato K, Tsuchida H. Polysaccharides from Agaricus blazei stimulat lymphocyte Tcell subsets in mice. Biosci Biotechnol Biochem. 1998; 62(3):434-7.

47. Hashimoto T, Nonaka Y, Minato K, Kawakami S, Mizuno M, Fukuda I, et al. Suppressive effect of polysaccharides from the edible and medicinal mushrooms, Lentinus edodes and Agaricus blazei, on the expression of cytochrome P450s in mice. Biosci Biotechnol Biochem. 2002;66(7):1610-14.

48. Wasser SP. Medicinal Mushrooms as a source of antitumor and immunomodulating polysaccharides. Appl Microbiol Biotechnol. 2002;60:258-74.

49. Júnior JF. Agaricus: cogumelo brasileiro com alguns efeitos anti-câncer. J Biomolec Med Free Rad. 1999;5(2): 36-7.

50. Sorimachi K, Akimoto K, Ikehara Y, Inafuku K, Okubo A, Yamazaki S. Secretion of TNF alfa, IL-8 and nitric oxid by macrophages activated with Agaricus Blazei Murril fraction in vitro. Cell Struct Funct. 2001 Apr;26(2):103-8.

51. Novaes MRCG, Novaes LCG, editors. Evaluation of acute toxicity of edible mushroom Agaricus sylvaticus on rats. Proceedings of the 27th International Congress of ESPEN and Clinical Nutrition; 2005 Aug 27-30; Brussels, Belgium: Elsevier 2005.

52. Novaes MRCG, Fortes RC, editors. Alterations on the metabolism of lipids in post-surgery patients with colorectal cancer supplemented with fungus Agaricus sylvaticus. Proceedings of the 27th International Congress of ESPEN and Clinical Nutrition; 2005 Aug 27-30; Brussels, Belgium: Elsevier 2005.

53. Liao ML, Zhao JM. The II stage clinical tests of PSP in the treatment of lung cancer. Apud.: Yang QY, Kwok CY. Proceedings of PSP International Symposium. Shangai, China: Fudan University Press, 1993, p.243-256.

54. Tsang KW, Lam CL, Yan C, Mak JC, Ooi GC, Ho JC, et al. Coriolus versicolor polysaccharide peptide slows progression of advanced non-small cell lung cancer. Respir Med. 2003;97(6):618-24.

55. Nakazato H, Koike A, Saji S, Ogawa N, Sakamoto J. Efficacy of immunochemotherapy as adjuvant treatment after curative resection of gastric cancer. Lancet. 1994;343:1122-26.

56. Shimizu S, Kitada H, Yokota H, Yamakawa J, Murayama T, Izumi $\mathrm{H}$, et al. Activation of the alternative complement pathway by Agaricus blazei Murril. Phytomedicine. 2002;9(6):536-45.

57. Yang QY, KwokCY.Proceedings of PSP International Symposium. Shangai: Fudan University Press; 1993. p. 232-40.

58. Dolby V. An extract from maitake mushroom is an important anti-cancer. Better Nutr. 1997;59(8):38.

59. Novaes MRCG, Fortes RC, editors. Effects of nutritional supplementation with Agaricus sylvaticus (Cogumelo do Sol) in post-surgery patients with adenocarcinoma of gross intestine. Proceedings of the 27th International Congress of ESPEN and Clinical Nutrition; 2005 Aug 27-30; Brussels, Belgium: Elsevier 2005. 